\title{
Eine Frage der Reaktion
}

Liebe Leserin, lieber Leser,

eine im Mai 2021 veröffentlichte Studie des Fraunhofer IES sagte für Deutschland eine Anzahl von etwa vier Millionen Elektrofahrzeugen (BEVs) in 2030 voraus, was zu einem zusätzlichen Strombedarf von etwa 11,6 Terawattstunden führe. Zwar sehen die meisten Energieversorger offiziell das Laden der immer schneller steigenden Zahl an Elektrofahrzeugen auch künftig als wenig problematisch und lösbar an, da ja nicht alle gleichzeitig am Netz hängen. Sicherlich erfordert dies aber ein möglichst perfektes Zusammenspiel von Erzeugung, Verbrauch, Speicherung und ein intelligentes, hochreaktives Netz, das so heute noch nicht existiert.

Prinzipiell bringt eine kritische Masse an Elektrofahrzeugen steigende Anforderungen an das Netz und seine Regelungstechnik mit sich: Dies resultiert aus dem Anteil an volatilen erneuerbaren Energien im Energiemix, deren Menge - um die Anforderungen an die grüne, Fridays-forFuture-taugliche All-electric-Society zu erfüllen - zudem extrem schnell gesteigert werden muss. Verstärkt werden die Effekte auf das Netz durch die bevorstehende Abschaltung der Kohlekraftwerke und damit Wegfall ihrer stabilisierenden Generatoren. Dies ist lösbar, erfordert aber einigen Aufwand.

Am einfachsten geschieht das, indem die Speicher von Elektrofahrzeugen mit einbezogen werden. Denn ohne einige sehr große Systeme oder sehr viele kleine Puffer wird eine Stabilisierung des Netzes nicht gelingen. Insofern kommt bidirektionalem Laden als wichtigem Ausgleichselement eine große Bedeutung zu. Dafür genutzt werden können neben BEVs auch in gewissem Ausmaß Plug-in-Hybride. Spannend wird das allerdings durch die schiere Masse an heterogenen beteiligten Speichersystemen, die es inklusive aktueller Verfügbarkeit, Zustand und
Eigenschaft zu koordinieren gilt: Zusätzlich werden ja noch weitere Konzepte von Schwerkraftspeichern bis zu Großbatterien untersucht.

Interessant wird es auch sein zu sehen, wie sich die (grüne) Energieerzeugung im Spannungsfeld zwischen Minimalerzeugung und Maximalverbrauch einpendelt. Zumal beim Puffern der ach so effizienten, sauberen elektrischen Energie dann doch die Verluste beim Wandeln ein Thema werden. Im Bereich von Terawattstunden wird dies augenfällig: Selbst wenn nur einige Prozent davon bidirektional fließen, kommt dem Kampf gegen jedes Zehntelprozent Verschwendung in diesen Größenordnungen Bedeutung zu. Dennoch ist es unabdingbar, die nötigen häufigen Regelaktionen um die 50 Hertz zu priorisieren, um das Netz stabil zu halten. Der Interessenskonflikt zwischen Netzstabilität und den dafür in Kauf zu nehmenden kumulierten Verlusten muss insofern in die Diskussion rund um die Energiewende mit einbezogen werden.

Viel Spaß bei der Lektüre des Hefts.

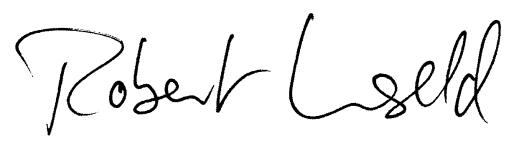

Robert Unseld Verantwortlicher Redakteur

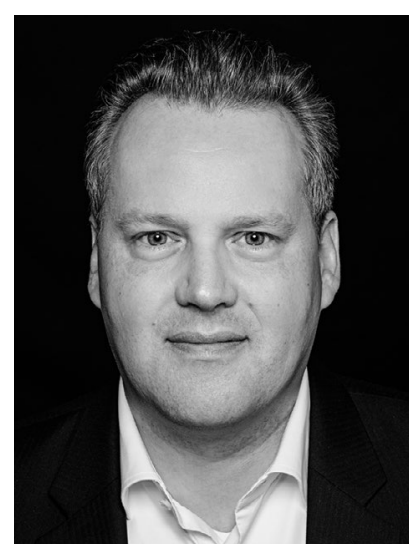

WATCH OUR VIDEO AND GET TO LEARN MORE: 